\title{
Availability and Nutritional Adequacy of Plant-Based Items in Public High School Lunch Menus
}

\author{
Abigail Singstock ${ }^{1}$ and Kelsey Randall ${ }^{1 \#}$ \\ ${ }^{1}$ Sycamore High School, Montgomery, OH, USA \\ \#Advisor
}

$\underline{\text { ABSTRACT }}$

Despite a growing body of research that indicates the health benefits of eating less meat and more plant-based foods, there is limited research on the actual availability of these plant-based options in high schools regulated by the National School Lunch Program (NSLP). This study aims to explore the availability of plant-based food items in public Cincinnati high schools enrolled in the NSLP and to determine whether the nutritional profiles of available plant-based options meet the caloric needs of high school students seeking a plant-based diet. The plant-based diet index (PDI) was used to provide a metric for whether a food item is absent of any animal products and can be further divided into healthy (hPDI) and unhealthy (uPDI) options. 361 lunch options from 8 Cincinnati public high schools were categorized into four food groups: Entrees, Sides, Vegetables and Fruits. It was found that there is a considerable scarcity of plant-based entree items, which account for only $1.3 \%$ of all entrees. Consequently, students following a plant-based diet would be unable to meet their caloric requirements at $75 \%$ of the studied schools (174 of $\sim 800$ recommended calories), and students following a healthful plant-based diet could not meet their caloric needs at any of the studied schools (131 of $\sim 800$ recommended calories). This may leave students who are reliant on the NSLP unable to obtain the health benefits of a plant-based diet, which would have disproportionate consequences for poorer students.

\section{Introduction}

Since the inception of the National School Lunch Program (NSLP), the United States Department of Agriculture (USDA) has provided dietary guidelines aimed at improving nutritional intake for America's children (NSLP, 2021). Originally designed to combat hunger, the NSLP is now tasked with providing free and reduced-price meals that address the growing concerns of obesity and other chronic diseases affecting children and adolescents. Serving 30.4 million meals per day, the impact of the NSLP on the current and future wellness of American children cannot be understated. Approximately 100,000 public schools participate, making the NSLP well positioned to promote healthy eating habits and attitudes. Research has shown that student access to healthy plant-based foods can establish lifelong dietary patterns that lead to improved health outcomes (Kim et al., 2019; Satija et al., 2016; Patel et al., 2020). In contrast, meat-based diets have been linked to acute and chronic diseases such as obesity, type 2 diabetes, cardiovascular disease, cancer, and an increase in all-cause mortality (Patel et al., 2020). There is significant importance to whether plant-based items are included as options for students to choose at lunch considering the health benefits, yet no prior research has been conducted revolving around this topic of inquiry.

\section{Gap in Research}

Multiple peer-reviewed studies have demonstrated a positive correlation between increased intake of plant-based foods and decreased incidence of chronic diseases; however, there is limited research on the extent to which plantbased foods are available in high schools and how those food items align with the Plant-Based Diet Index (PDI). The 
purpose of this study is to understand the availability of plant-based options available for students to consume and whether students would be able to sustain a plant-based diet with the given options provided by their school. Considering that schools implement a high availability of plant-based items into their menus, this would provide students with increased health benefits and reduced risks of developing many chronic diseases.

\section{Literature Review}

\section{Search Strategies}

Sources were found with various online databases for peer reviewed articles (e.g., Google Scholar) to ensure source credibility. The keywords used while conducting research were: National School Lunch Program (NSLP), United States Department of Agriculture (USDA), plant-based, plant-based diet, vegan, school menus, childhood obesity, meat alternatives, Plant-Based Diet Index (PDI), Cardiovascular Disease, and Type Two Diabetes.

\section{The Impact of the National School Lunch Program}

The impact of the National School Lunch Program (NSLP) on the current and future wellness of American children cannot be understated. In 2010, the NSLP was redefined to address inadequate dietary intake, inactive lifestyles, and the growing health concerns of America's children. The Healthy Hunger-Free Kids Act of 2010 identified the need for a more healthful diet which included whole grains, vegetables, fruits, lean proteins, and a reduction of added sugars, sodium, and refined grains (USDA, 2020). In an effort to better meet dietary needs and provide diversification in menu options, in 2012 the USDA approved the reimbursement or "credit" to school districts serving soy and tofu as long as these substitutes were easily recognized as meat alternatives. Schools choosing to offer meat alternatives were guaranteed federal financial reimbursement under this addendum (USDA, 2016). Reimbursement credit for tempeh, enriched protein pasta, and legumes followed in 2019 (USDA, 2019). Current USDA recommendations encourage a lifespan approach to the reduction of saturated fat and red meat, as well as suggest that American's eat more plantbased foods (USDA, 2020).

\section{The Plant-Based Diet Index}

Plant-based diets encompass a wide variety of dietary patterns; however, they generally aim to maximize consumption of plant foods while minimizing or eliminating the intake of animal-based foods, including dairy and eggs. These diets include fruits and vegetables as well as nuts, seeds, legumes, and many whole grains. Created by researchers at Harvard's TH Chan's School of Public Health, the Plant-Based Diet Index (PDI) assigns a reverse (negative) score to any food items derived from or including animal products and assigns a positive score to foods that are entirely derived from plants. Eighteen food groups (Figure 1) are present in the index and are used to identify and score food items as plant-based. It also further delineates foods into healthful plant-based (hPDI) and unhealthful plant-based (uPDI). For example, fruit juices are plant-based and have a positive PDI score; however, they contain high amounts of simple sugars, which increase risks of Type 2 Diabetes, Obesity, and Coronary Heart Disease (Kim et al., 2019). Fruit juices would therefore be classified with a positive uPDI score and a negative hPDI score. The food groups defined in the Plant-Based Index were used as a credible reference for identifying and analyzing the menu items collected in this study. Permission to use the Plant-based Diet Index was granted by Megu Baden (Appendix A). 


\begin{tabular}{|c|c|c|c|c|}
\hline & & PDI & hPDI & uPDI \\
\hline \multicolumn{5}{|c|}{ Plant Food Groups } \\
\hline \multicolumn{5}{|l|}{ Healthy } \\
\hline Whole grains & $\begin{array}{l}\text { Whole grain breakfast cereal, other cooked breakfast cereal, cooked oatmeal, } \\
\text { dark bread, brown rice, other grains, bran, wheat germ, popcom }\end{array}$ & Positive scores & Positive scores & Reverse scores \\
\hline Fruits & $\begin{array}{l}\text { Raisins or grapes, prunes, bananas, cantaloupe, watermelon, fresh apples or } \\
\text { pears, oranges, grapefruit, strawberries, blueberries, peaches or apricots or } \\
\text { plums }\end{array}$ & Positive scores & Positive scores & Reverse scores \\
\hline Vegetables & $\begin{array}{l}\text { Tomatoes, tomato juice, tomato sauce, broccoli, cabbage, cauliflower, } \\
\text { brussels sprouts, carrots, mixed vegetables, yellow or winter squash, } \\
\text { eggplant or zucchini, yams or sweet potatoes, spinach cooked, spinach } \\
\text { raw, kale or mustard or chard greens, iceberg or head lettuce, romaine or } \\
\text { leaf lettuce, celery, mushrooms, beets, alfalfa sprouts, garlic, corn }\end{array}$ & Positive scores & Positive scores & Reverse scores \\
\hline Nuts & Nuts, peanut butter & Positive scores & Positive scores & Reverse scores \\
\hline Legumes & String beans, tofu or soybeans, beans or lentils, peas or lima beans & Positive scores & Positive scores & Reverse scores \\
\hline Vegetable oils & Oil-based salad dressing, vegetable oil used for cooking & Positive scores & Positive scores & Reverse scores \\
\hline Tea and coffee & Tea, coffee, decaffeinated coffee & Positive scores & Positive scores & Reverse scores \\
\hline \multicolumn{5}{|l|}{ Less healthy } \\
\hline Fruit juices & $\begin{array}{l}\text { Apple cider (nonalcoholic) or juice, orange juice, grapefruit juice, other fruit } \\
\text { juice }\end{array}$ & Positive scores & Reverse scores & Positive scores \\
\hline Refined grains & $\begin{array}{l}\text { Refined grain breakfast cereal, white bread, English muffins or bagels or rolls, } \\
\text { muffins or biscuits, white rice, pancakes or waffles, crackers, pasta }\end{array}$ & Positive scores & Reverse scores & Positive scores \\
\hline Potatoes & French fries, baked or mashed potatoes, potato or corn chips & Positive scores & Reverse scores & Positive scores \\
\hline Sugar sweetened beverages & $\begin{array}{l}\text { Colas with caffeine and sugar, colas without caffeine but with sugar, other } \\
\text { carbonated beverages with sugar, noncarbonated fruit drinks with sugar }\end{array}$ & Positive scores & Reverse scores & Positive scores \\
\hline Sweets and desserts & $\begin{array}{l}\text { Chocolates, candy bars, candy without chocolate, cookies (home-baked and } \\
\text { ready-made), brownies, doughnuts, cake (home-baked and ready-made), } \\
\text { sweet roll (home-baked and ready-made), pie (home-baked and ready- } \\
\text { made), jams or jellies or preserves or syrup or honey }\end{array}$ & Positive scores & Reverse scores & Positive scores \\
\hline \multicolumn{5}{|c|}{ Animal Food Groups } \\
\hline Animal fat & Butter added to food, butter or lard used for cooking & Reverse scores & Reverse scores & Reverse scores \\
\hline Dairy & $\begin{array}{l}\text { Skim low fat milk, whole milk, cream, sour cream, sherbet, ice cream, yogurt, } \\
\text { cottage or ricotta cheese, cream cheese, other cheese }\end{array}$ & Reverse scores & Reverse scores & Reverse scores \\
\hline Egg & Eggs & Reverse scores & Reverse scores & Reverse scores \\
\hline Fish or seafood & Canned tuna, dark meat fish, other fish, shrimp or lobster or scallops & Reverse scores & Reverse scores & Reverse scores \\
\hline Meat & $\begin{array}{l}\text { Chicken or turkey with skin, chicken or turkey without skin, bacon, hot dogs, } \\
\text { processed meats, liver, hamburger, beef or pork or lamb mixed dish, beef } \\
\text { or pork or lamb main dish }\end{array}$ & Reverse scores & Reverse scores & Reverse scores \\
\hline $\begin{array}{l}\text { Miscellaneous animal-based } \\
\text { foods }\end{array}$ & Pizza, chowder or cream soup, mayonnaise or other creamy salad dressing & Reverse scores & Reverse scores & Reverse scores \\
\hline
\end{tabular}

Figure 1: The Plant-based Diet Index

\section{Dietary Intake and Obesity}

One in every six children in the United States is obese (CDC, 2018). Obesity, defined as a body mass index (BMI) at or above the 95th percentile, is a contributing factor to chronic disease and decreased life expectancy (Lee \& Yoon, 2018). According to the Center for Disease Control (CDC), the prevalence of obesity in children aged $12-19$ is $20.6 \%$ (CDC, 2018). In a study published in The New England Journal of Medicine, researchers developed a growth trajectory simulation model to track current trends of obesity from childhood into adulthood and concluded that based on current trends, $57 \%$ of children who are currently between the ages of 2 and 19 would be obese at the age of 35 (Ward et al., 2017). Research has shown there are many contributing factors to obesity and concluded that establishing healthful eating patterns as a preventative weight management strategy was shown most effective when begun as early as possible (Lee \& Yoon, 2018). Childhood obesity continues to rise, and according to Li and Hooker, students eligible for the NSLP have a $4.5 \%$ higher incidence of obesity than those who attended public schools but do not participate in the NSLP (Li \& Hooker, 2010). Schanzenbach (2009) found that children eligible for free and reduced lunches through the NSLP were more often obese than those children eating a lunch prepared from home. After controlling for other possible contributing factors, Schanzenbach concluded that students who are eligible for reduced lunch have the highest incidence of obesity. It has been shown that there is a positive relationship between meat consumption and obesity, whereas vegetarian and vegan eating habits have lower rates of obesity. Wang and Beydoun (2009) used 
nationally representative data from the National Health and Nutrition Examination Study to study meat consumption and concluded that those who had high meat consumption were $27 \%$ more likely to be obese. In contrast, epidemiologic studies have shown vegetarian and vegan children to have lower rates of being overweight or obese than those who are nonvegetarian (Sabate \& Wien, 2010). With a primary goal of the NSLP to address inadequate dietary intake and provide more healthful food choices, the availability of plant-based foods for weight management warrants further thought.

\section{Dietary Intake and Diabetes}

Being overweight is a significant risk factor for Type 2 Diabetes. $85 \%$ of overweight adults have Type 2 diabetes, and the prevalence is on the rise in youth (CDC). In a peer-reviewed study using the Plant-Based Diet Index, researchers concluded that high-quality plant-based foods are associated with a substantially lower risk of developing Type 2 diabetes in adults (Satija et al., 2016). They further concluded that consumption of a plant-based diet high in healthy plant foods reduced diabetes risk by $34 \%$, while an unhealthy plant-based diet was associated with a $16 \%$ increased diabetes risk (Satija et al., 2016). This distinction identifies that not all plant-based food items are considered healthy. According to the CDC (2020), the incidence of Type 2 diabetes in youths under 20 years old increased in the United States between 2002-2015 at a rate of 4.8\% per year. In addition, youth onset of Type 2 diabetes progresses more rapidly than adult-onset diabetes and has been shown to remain poorly controlled into adulthood (Ke et al., 2020) meaning if prevented earlier on in high school with a plant-based diet, it could reduce the risk of developing adulthood Type 2 diabetes.

\section{Dietary Intake and Coronary Heart Disease}

Coronary Heart Disease (CHD) is the leading cause of death in the United States, killing over 650,000 Americans each year (CDC, 2020). Continued research in the field of nutrition has found a direct correlation between consuming plant-based foods and improved health. Through a systematic collection of data over decades, researchers at Harvard Medical School concluded that greater intake of plant-based foods richer in healthier plant foods is associated with a substantially lower risk of developing coronary heart disease. While a plant-based diet that includes less-healthy plant foods is associated with a higher risk in developing coronary heart disease (Satija et al., 2017). This study took a comprehensive look at diet, lifestyle, and medical history, compiling that information into multiple datasets, including the PDI, hPDI and uPDI datasets as described in Figure 1. A study published in the Journal of the American Heart Association focused its data collection on those at risk for cardiovascular disease. Kim and others (2019) reported daily food intake of adult men and women collected over two years and assigned scores based on the three categories: PDI, hPDI, and uPDI. This categorization excluded animal-based products and focused the study on the health risks associated with unhealthy plant-based foods (uPDI) like refined carbohydrates and simple sugars. Although items in the uPDI are not animal-based products, they are often high in sugar and low in nutrient-dense calories, which are often associated with obesity, diabetes, and cardiovascular disease (Kim et al., 2019). Their study concluded that a "higher adherence to a healthy plant-based diet index was associated with a $19 \%$ and $11 \%$ lower risk of cardiovascular disease mortality and all-cause mortality, respectively" (Kim et al., 2019). Researchers have found that following a healthful plant-based diet is the only dietary pattern that reduces coronary artery disease (Kahleova, Levin, \& Barnard, 2018).

Summary 
The health benefits and reduced health risks revolving around plant-based diets, further amplifies the importance of searching for the availability of plant-based lunch menu items within public high schools. Considering youth development of chronic diseases leads to increased risk of developing other diseases and mortality within adulthood, it provides even more emphasis on my research question and topic of inquiry.

\section{Design and Methodology}

\section{Study Design}

This study is aimed to explore the availability of plant-based lunch menu options at public high schools enrolled in the NSLP in the Cincinnati area. The purpose of this study is to explore to what extent plant-based food items are available and if the nutritional components of those items meet the caloric needs of high school students seeking a vegan or other plant-based diet. This is important considering the reduced risks of developing chronic health conditions by the increased consumption of plant-based foods.

A three-part study was conducted by using a content-analysis for the method design. A content analysis is a research method used to evaluate the presence and frequency of specific words, themes, and concepts within a given data set (Columbia Public Health, 2021). The content-analysis enabled direct analysis of the availability of plant-based lunch menu items in gathered lunch menus. This research method was chosen to be used in this study because it positions the data to be analyzed both quantitatively and qualitatively. This facilitated a more in-depth understanding of the availability of plant-based items. The three-part study included: a search for keywords, assignment of PDI values, and assessment of caloric values (Figure 2).

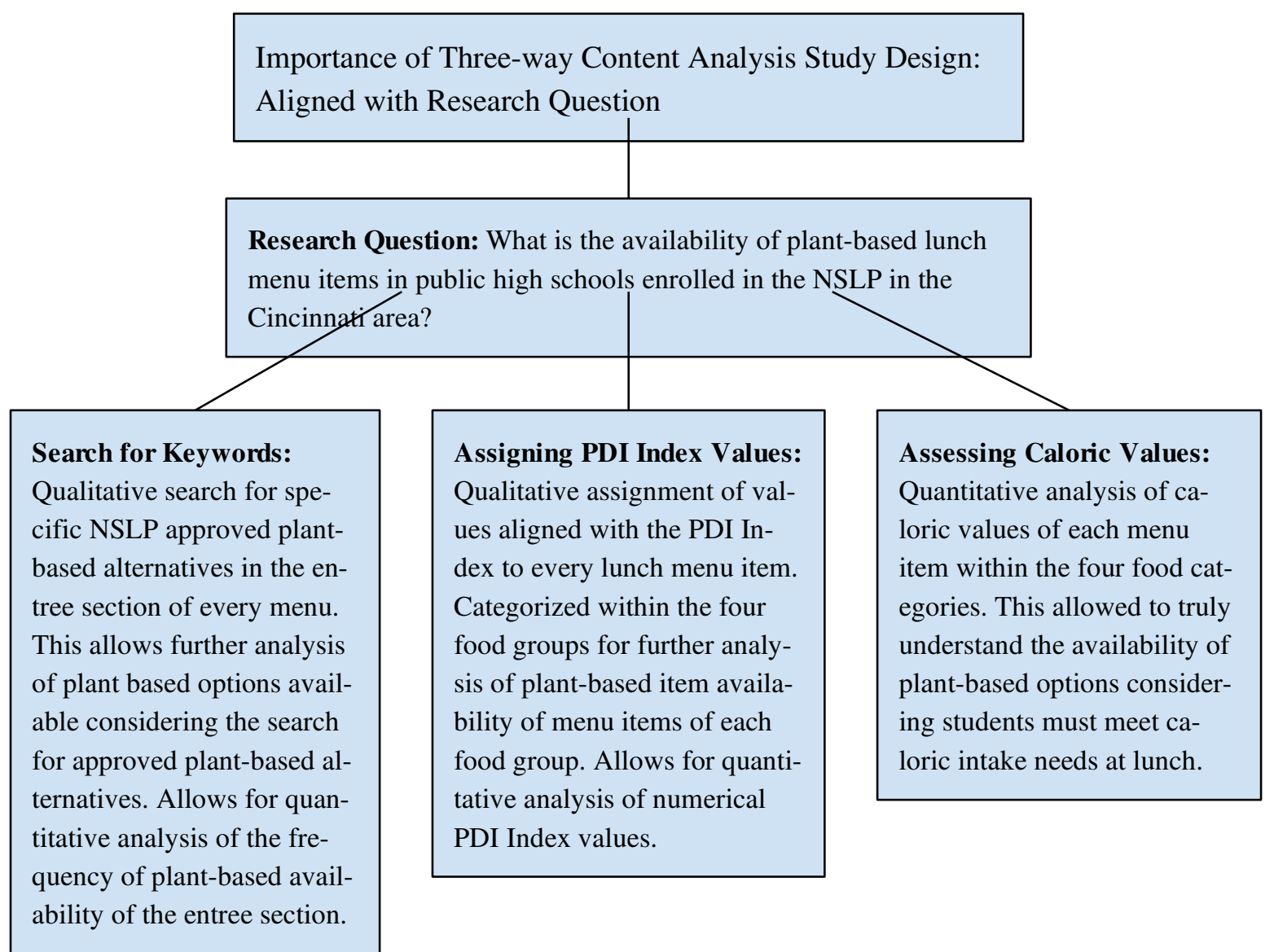


Figure 2. The 3-part Content Analysis in Alignment with the Research Question

In order to assess the availability of plant-based foods, I analyzed the menu items available over the course of one month, November 2020, at eight local Cincinnati high schools enrolled in the NSLP. High schools were chosen considering they represent youth on the verge of adulthood meaning if plant-based diets were implemented while students are in high school, it could prevent the risk of developing chronic diseases as noted in the literature review. Parameters for inclusion were narrowed to 1) public high schools in Cincinnati and surrounding suburbs serving over 1,000 students per day, 2) offered published online menus on their public school website and 3) participated in the National School Lunch Program. Participation in NSLP assured food provided met USDA and National School Lunch Program (NSLP) guidelines. Choosing public high schools in the NSLP allows for my research to apply to more schools not included within the study that are also enrolled in the NSLP. Choosing the Cincinnati area represents many different socioeconomic statuses and is highly diverse, which makes the findings of this study translatable to other diverse cities. Lastly, schools serving over 1,000 students allows my research to apply to a larger population of students.

Two school districts were excluded due to Covid-19 protocol which limited the lunch menu to grab and go boxed items; therefore not having a menu for analysis. Leaving eight public high schools representing over 15,000 students meeting the criteria of inclusion for this study.

After schools were selected, menus from each school district were collected via their public website and the interactive online menu application, School Cafe, provided daily nutritional information for each entree, side, vegetable, and fruit available. For school districts not using the School Cafe application, menus were printed from the school districts' website. Where limited nutritional information was provided by the school website, the website food calculator WebMD was used to estimate the nutritional information needed to complete the data analysis (WebMD 2015). When entering menu items on WebMD, items were entered as a single serving size for students in grades 9-12 based on NSLP guidelines.

The sorting and categorization of all 361 menu items was performed using Google Spreadsheets. All menu items were manually entered into a spreadsheet which separated each school using different tabs to ensure organization. Nutritional data (calories, fat, saturated fat, fiber, sugar, and protein) was entered also manually into a spreadsheet and a dataset was generated for statistical analysis. Manual entry allowed the data to be within the same format, enabling easier analysis of menu items and a lower chance of mistake to occur. Lastly, the menu items were categorized into four food categories: Entrees, Sides, Vegetables and Fruits. These are the four categories students are required to take a menu item from according to the NSLP to qualify for reduced lunch prices. Again, the NSLP requires a student to take only one item from each category. Analyzing menu items within these four categories allows further depth and understanding of the availability of plant-based options within those food categories.

\section{Search for Keywords}

The NSLP provides financial reimbursement to school districts for meat or meat alternatives provided in the lunch meal. I searched each schools entree food group for the following keywords "tofu", "tempeh", "enriched plant-based pasta", "almond butter", "cashew nut butter", "reduced fat peanut butter", "peanut butter", "sesame seed butter", "soy nut butter", and "sunflower seed nut butter", "legumes", and "refried beans". These keywords contain the NSLP approved plant-based alternatives that can be included in entrees instead of meat products. The entrees were the only food category searched because this is the only category which qualifies to include meat alternatives by NSLP regulations. This search was done by using the search database function provided by Google Sheets. This function will show the amount of times the entered word appears throughout the entire database of menus from each school researched. This search was conducted to see the availability of meat alternatives that could be consumed by a student following a plant-based diet, thus aligning with my research question by looking for availability of plant-based alternatives. 


\section{Assigning PDI Index Values}

The content analysis of high school menus was coded to identify all menu items as Plant-Based (PDI), Healthful PlantBased (hPDI), and Unhealthful Plant-Based (uPDI). The plant-based diet index (PDI) is an index created by Harvard researchers, Satija, et al., and is an accepted standard in scientific articles. It was used as a reference for the analysis of highschool lunch menu items. I hand assigned PDI index values to every menu item based off of the PDI index (Figure 1). Each menu item received a score: positive or reverse. A positive score indicates the menu item qualifies for that value. For example, an apple receives a positive score for both PDI and hPDI considering it is plant-based and healthy plant-based according to the PDI Index. If an item received a reverse score, they did not qualify for inclusion within that value. An example includes a slice of pepperoni pizza. This item receives a reverse score from the PDI value considering its inclusion of multiple animal products (meat and cheese) but, since it does not meet criteria of inclusion for the PDI value, it scores reverse for both uPDI and hPDI values. Using the PDI Index allows each menu item to be analyzed based on whether it is considered plant-based which overall allows better understanding of the availability of plant-based lunch menu options. Permission to use the Plant-based Diet Index was granted by Megu Baden (Appendix A).

\section{Assessing Caloric Values}

Caloric values were collected from November 2020 menus by reviewing school districts' public websites for menu and nutritional information. Recalling that daily menu items offered were categorized by entree, side, vegetable, and fruit, this provided further assessment of calories within those categories. Milk was excluded from the study because, although milk is provided by the NSLP, currently no milk substitutes are approved; therefore, milk was easily categorized as not plant-based and not included in this study. Nutritional information per serving of each menu item was manually entered for calories, fat, sodium, carbohydrates, sugars, dietary fiber, and protein. The purpose of recording this data was to identify if a student seeking a plant-based diet could have their caloric needs met from the available lunch options. As previously mentioned, caloric recommendations for students in grades 9-12 would be 750-850 calories for the lunch meal. If a student cannot meet their caloric intake needs through the means of a plant-based diet provided by their school's lunch options, that is considered unsustainable. They would have to attain calories from another source, whether that be packing lunch or consuming items with animal products.

\section{Results}

\section{Search for Keywords: Findings and Discussion}

The search algorithm concluded that only one entree menu item was found using the specific following keywords "tofu", "tempeh", "enriched plant-based pasta", "almond butter", "cashew nut butter", "reduced fat peanut butter", "peanut butter", "sesame seed butter", "soy nut butter", "sunflower seed nut butter", "legumes", and "refried beans" from any of the schools measured. Out of 153 entree items, only two had the keyword "peanut butter" (Table 1). The menu item found was a Smuckers Peanut Butter and Grape Jelly Sandwich, which was offered at two of the eight schools. Six of the eight schools (75\%) did not offer any of the plant-based alternative entrees. Even though plantbased alternatives were approved by the NSLP beginning in 2012, availability was extremely limited and a student following a plant-based diet at six of the eight schools would not have access to plant-based entree options on any school day of the month. This shows the need for more implementation of plant-based alternatives to the entree section of public high schools enrolled in the NSLP.

Table 1. Total Count of Food Groups aligned with PDI Index 


\begin{tabular}{|l|l|l|l|l|l|}
\hline & n (count) & $\begin{array}{l}\text { \# of animal prod- } \\
\text { uct }\end{array}$ & \# of PDI values & \# of uPDI Values & \# of hPDI Values \\
\hline Total Entrees & 153 & 151 & 2 & 2 & 0 \\
\hline Total Sides & 50 & 20 & 30 & 15 & 15 \\
\hline Total Vegetables & 72 & 3 & 69 & 1 & 68 \\
\hline Total Fruits & 86 & 0 & 86 & 26 & 60 \\
\hline
\end{tabular}

\section{PDI Index: Findings and Discussion}

After assigning PDI values, it was found that the two food categories "Vegetables" and "Fruits" received the highest percentage of positive PDI values, at $96 \%$ and 100\%, respectively (Figure 3). Of those vegetables considered plantbased, 99\% received a positive hPDI score (Tables 1 and 2). The inclusion of ranch dressing and cheese with vegetables was the $4 \%$ exception in this category. Particular attention to sugar content in fruit juices and fruit cups, resulted in only $70 \%$ of the available fruits to be considered as hPDI. $60 \%$ of the "Sides" were found to be plant-based; however, only half of those menu items were considered hPDI. Sides that were fried and/or over processed such as french fries and white rice were found to be unhealthy and represented $50 \%$ of these plant-based side options. The final category analyzed and of significant importance to this study was the availability of plant-based entrees. Of the 153 entrees available, 1.3\% were considered plant-based (Figure 3). The Smuckers Peanut Butter and Grape Jelly Sandwich, as found in the search earlier, was the only item found that was plant-based. Although plant-based, this item was assigned uPDI for multiple reasons. It was found that this one item had the highest number of calories (600), highest number of fat (34g), highest number of carbohydrates $(64 \mathrm{~g})$, and the highest number of sugar calories $(29 \mathrm{~g})$ of all entrees. In regards to the rest of the entrees, the extremely low percentage of plant-based entrees shows plantbased students would lack an entree item in their meals. In addition, sides included $60 \%$ plant-based options, which allows for a $40 \%$ chance that a plant-based student may not receive a side item in their meal as well. If students are not receiving an entree or a side, they are not receiving a full lunch meal according to the NSLP recommendations of receiving a food item from each food category. In conclusion, the results of this study have shown a critical lack of plant-based items, particularly for entree items, for a student seeking a plant-based diet.

\section{Percentage of Plant Based Items from each Food Category}

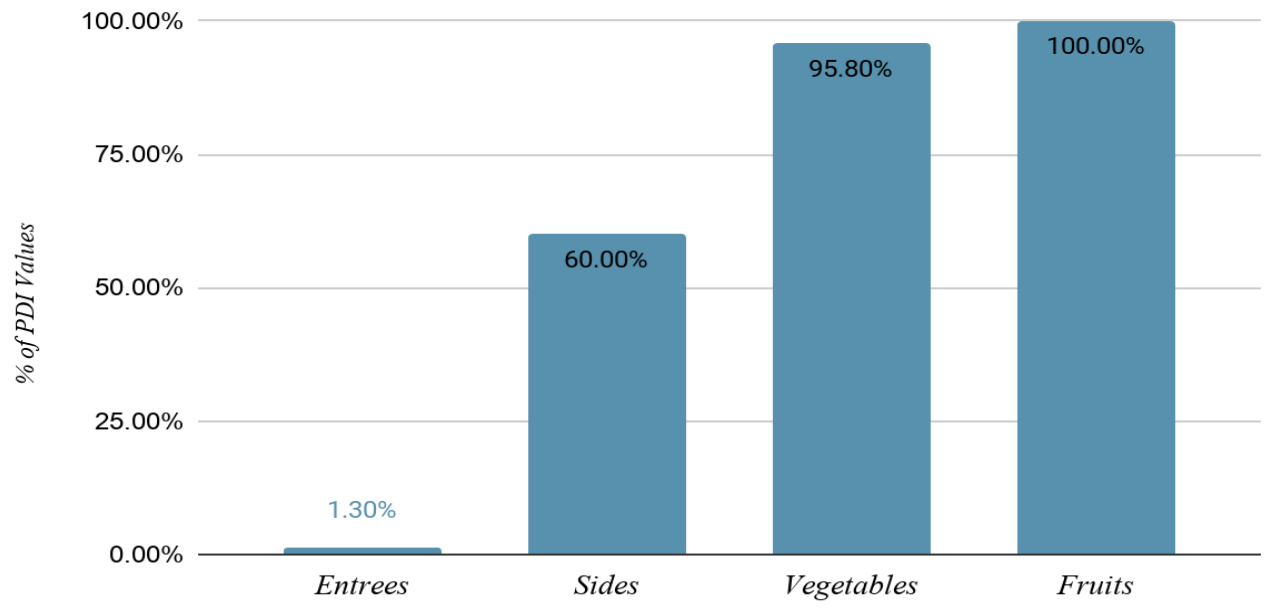


Figure 3. Plant-Based Items Available in all Four Food Categories

Table 2. Percentage of PDI Index Scores aligned with Each Food Category

\begin{tabular}{|l|l|l|l|}
\hline & Percentage of PDI scores & Percentage of hPDI scores & Percentage of uPDI scores \\
\hline Entrees & $1.3 \%$ & $0 \%$ & $100 \%$ \\
\hline Sides & $60 \%$ & $50 \%$ & $50 \%$ \\
\hline Vegetables & $96 \%$ & $99 \%$ & $1 \%$ \\
\hline Fruits & $100 \%$ & $70 \%$ & $30 \%$ \\
\hline
\end{tabular}

\section{Caloric Values: Findings and Discussion}

The average calories for each of the four food groups was calculated and summarized. The average for entrees was 350 calories, the average for sides was 138 calories, the average for vegetables was 52 calories and the average for fruits was 68 calories. The data shows that a lunch entree is needed to attain the $750-850$ calories recommended by the NSLP for students in grades 9-12. Noting back to the low availability of plant-based entrees and more moderate availability of plant-based sides, some students would not be able to include an entree or side within their meal if they follow a plant-based diet. Without entrees and sides, a plant-based student is receiving a significantly lower caloric intake at lunch compared to that of a student not following a plant-based diet. That would be unsustainable for a student to maintain and they would need to find alternative calorie sources, which would mean either bringing food from home or eating entrees and sides that are not plant-based. The caloric values of food groups Vegetables and Fruits were considerably low with an average of 52 calories for vegetable items and 68 calories for fruit items. Although the Vegetable and Fruit food groups are highly available for plant-based students to consume, they do not provide the amount of calories needed to complete a lunch that fulfills the recommended calories from the USDA. If plant-based students are to consume lunch at their school, chances are very high they will not receive the recommended calories to be consumed by a high school student.

To better explain the relationship between the food categories and their PDI and caloric results, representative lunch plates were created (Figure 4). Each plate represents what a student would receive based on whether they were following a typical Western diet (one which includes animal products), an unhealthy plant-based diet, or a healthy plant-based diet. The plate template was taken from Google Images Public Domain (Appendix B). Two plates of uPDI were created to account for the two schools (Schools F and G) that provide one entree item (Smuckers Peanut Butter and Grape Jelly Sandwich) that a student following a uPDI diet could consume. The other six schools (A, B, $\mathrm{C}, \mathrm{D}, \mathrm{E}$, and $\mathrm{H}$ ) provided no entree items for either hPDI or uPDI diets. This is reflected in the two uPDI plates.

To create an accurate plate for each diet plan, food items from each food category (entree, side, vegetable, and fruit) were selected to create a more precise representation. For example, a student following a healthful plant-based diet could not consume any entrees represented by any of the schools from the study so their plate excluded a food item from the entree food category.

These plates are a hypothetical visual representation of what one who is following a specific diet could consume daily for lunch at school. It can be noted that not all schools provide these options for plant-based students and a student would need to take a non plant-based entree to receive this lunch considering to qualify for reimbursement, an entree is required. 
Considering Plate A, a student following a healthful plant-based diet does not consume the daily lunch caloric values recommended by the NSLP. This shows that one following a plant-based diet cannot eat the hPDI items for lunch alone in order to be fully sustained with a lunch designed for a high schooler by the NSLP. This particular student would need to find calories sourced elsewhere, either food from home or an entree including animal products. For a student following a Western based diet (one which consumes animal products) they meet the caloric intake recommended by the NSLP (Plate B). This is considering the inclusion of the two high caloric food categories: Entrees and Sides. This provides further emphasis that with an entree included within one's meal, it allows for the meal to meet NSLP caloric requirements.

For the unhealthy plant-based options at school F and G (Plate C) which included the one plant-based entree option (Uncrustables Peanut Butter and Grape Jelly Sandwich), students met the caloric intake recommendation provided by the NSLP. This further explains how if more plant-based entree options are implemented within schools, students following a plant-based diet could receive a full lunch meal.

For schools A, B, C, D, E, and H (Plate D) which did not provide students with plant-based entree options, students did not meet the recommended calories to be consumed during lunch by the NSLP. Considering schools F and $\mathrm{G}$ did with the inclusion of an entree, it can be realized the entree category is extremely important in allowing students a full lunch meal that meets caloric guidelines.

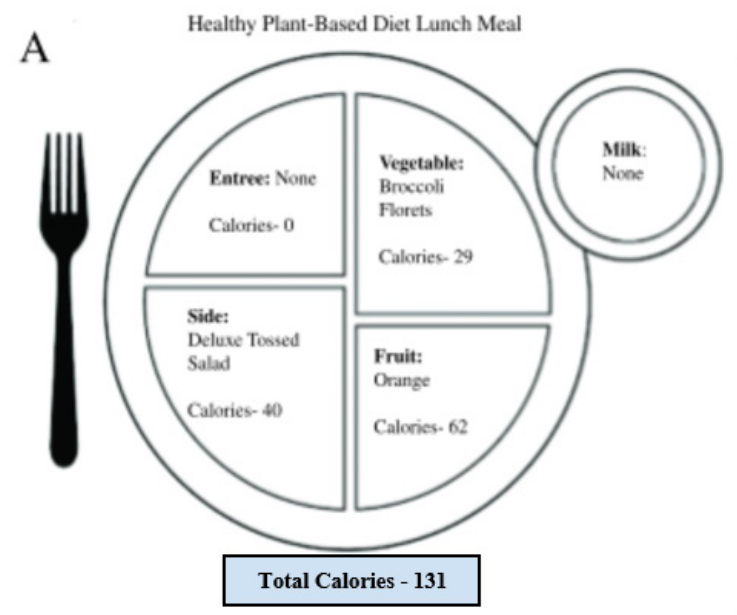

C

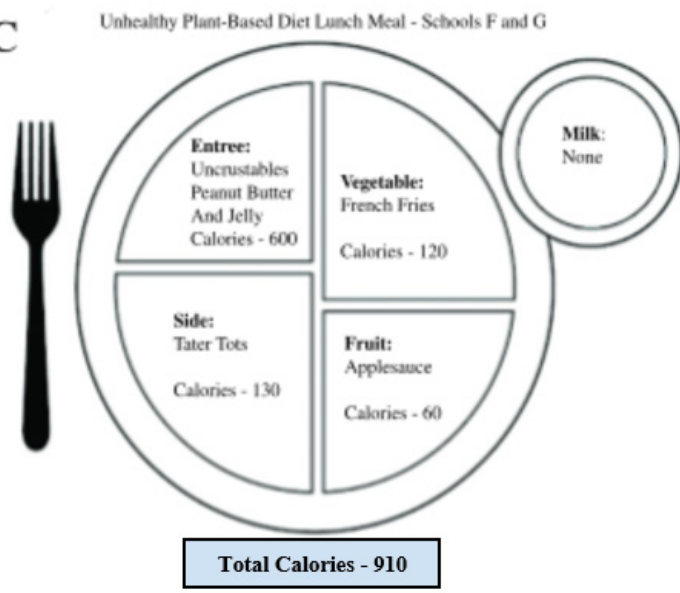

B

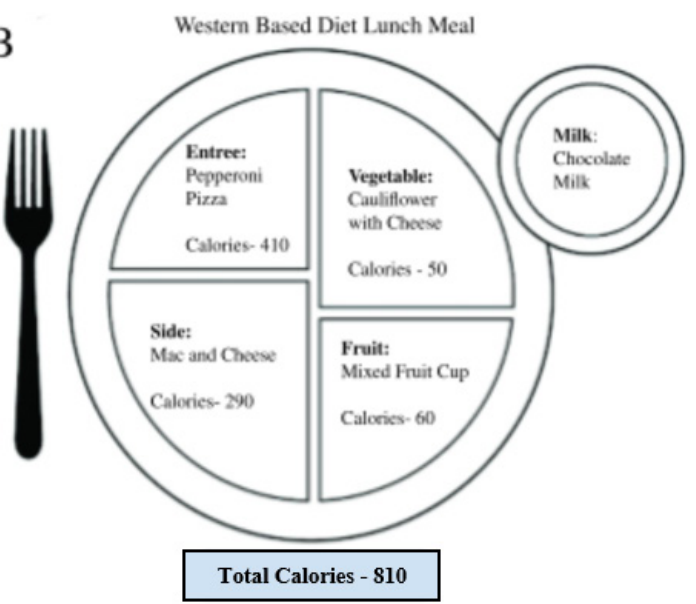

D

Unhealthy Plant-Based Lunch Meal - Schools A.B.C.D.E, and H

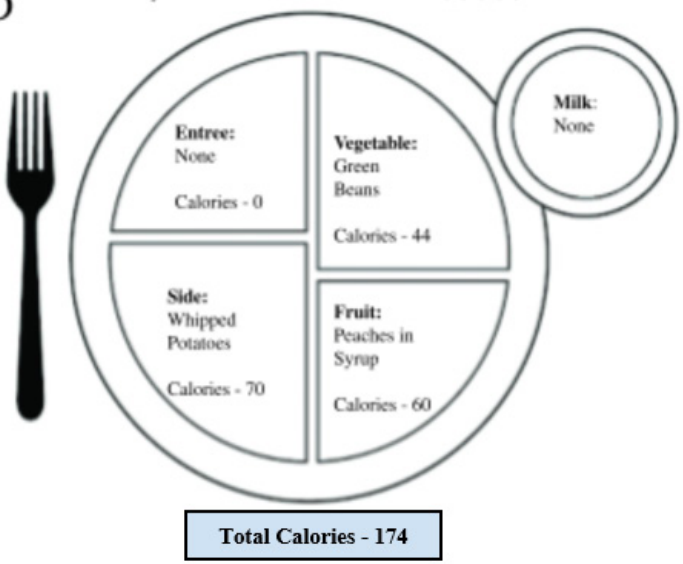

Figure 4. Hypothetical Lunch Plates of Varying Diets 


\section{Limitations}

This study had a limited sample size of 8 public schools. A larger sample could have led to more robust statistics and may have provided different results. Furthermore, all data entry was completed by hand meaning human error could have occurred and changed the results outcome. Another limitation would include only having access to published menus; meaning all data collected in the study was based off of what was publicly published by each school's website. This allows for the chance that additional items or a la carte options were available for purchase by students that could have been plant-based or not plant-based. Additionally, this study looked at availability of plant-based options observed from one month worth of published menus meaning changes in the menus after November 2020 were not analyzed or included in this study. Lastly, it is possible that COVID-19 service protocol could have resulted in menu changes meaning my results represent the school's response to the COVID-19 Pandemic and should not represent what options students receive within the typical school year.

\section{Conclusions}

Despite the impressive amount of data supporting consumption of plant-based foods for prevention and treatment of obesity and other diseases, the lack of availability of plant-based foods within public high schools should raise concern. Although federal approval of meat alternatives began as early as 2012, these results showed that a peanut butter and jelly sandwich is the only plant-based entree option available and its availability limited. Considering the low availability of plant-based entrees determined from the PDI Index, it can be concluded that the inclusion of more plantbased entrees, especially healthful plant-based entrees, is necessary for students following a plant-based diet.

With the direct correlation between consuming healthful plant-based foods and positive health outcomes, the NSLP has both the opportunity and obligation to recommend plant-based foods and incentivise school districts to provide at least one plant-based meal per day to students that would meet dietary recommendations for calories. Increased availability of plant-based options could have a rapid and positive impact, as researchers at the Cleveland Clinic found that in one month of eating a plant-based diet, children aged 9-18, lost weight and had "significant improvement in systolic blood pressure, body mass index (BMI), total cholesterol, and insulin levels" (Machnin et al., 2015). Considering all the health improvements associated with plant-based diets, it is extremely important that more plant-based foods (especially entrees) are implemented into public high school lunch menus so students can receive the health benefits and reduced risks of health conditions included in plant-based diets.

\section{Future Directions}

The results of this study have led to several questions which could direct future research. As previously mentioned, all nutritional data was collected from the school menus including levels of dietary fiber, protein, sugar, fats, and unsaturated fats. With my research question seeking the availability of plant-based foods, the nutritional profiles were only briefly reviewed and discussed but could be further analyzed to understand whether plant-based students are reaching other dietary nutritional standards.

Furthermore, with meat alternatives being available as early as 2012 , this study would suggest a possible disconnect between what is being approved and what is being served in the local high schools. I would further analyze why there is a low plant-based entree availability by conducting a survey of the Ohio Department of Education, school districts, and local food service personnel. I would ask them questions regarding the entree food options available to determine why alternatives are not present in high school lunch menus. 


\section{Acknowledgments}

I would like to express my gratitude and thanks to Kelsey Randall and my family for their guidance and continued support for my project.

\section{References}

CDC. (2021, April 5). Childhood Obesity Facts. Centers for Disease Control and Prevention. https://www.cdc.gov/obesity/data/childhood.html.

CDC. (2020, February 11). National Diabetes Statistics Report, 2020. Centers for Disease Control and Prevention. https://www.cdc.gov/diabetes/library/features/diabetes-stat-report.html.

CDC. (2020, February 11). Rates of New Diagnosed Cases of Type 1 and Type 2 Diabetes Continue to Rise Among Children, Teens. Centers for Disease Control and Prevention. https://www.cdc.gov/diabetes/research/reports/children-diabetes-rates-rise.html\#: :text=The $\% 20$ rate $\% 20$ of $\% 20$ new $\% 20$ cases,of $\% 20 \mathrm{CDC} \% 27 \mathrm{~s} \% 20 \mathrm{Morbid}$ ity $\% 20$ and $\% 20$ Mortality.

Columbia Public Health. (2021, May 7). Content Analysis. Public Health Methods. https://www.publichealth.columbia.edu/research/population-health-methods/content-analysis.

Freedman, D. S., Khan, L. K., Dietz, W. H., Srinivasan, S. R., \& Berenson, G. S. (2001, September 1). Relationship of Childhood Obesity to Coronary Heart Disease Risk Factors in Adulthood: The Bogalusa Heart Study. American Academy of Pediatrics. https://pediatrics.aappublications.org/content/108/3/712.

Galuska, D. A., Gunn, J. P., O'Connor, A. E., \& Petersen, R. (2018, November 31). Addressing Childhood Obesity for Type 2 Diabetes Prevention: Challenges and Opportunities. National Institutes of Health. https://www.ncbi.nlm.nih.gov/pmc/articles/PMC6243220/.

Kahleova, H., Levin, S., \& Barnard, N. D. (2018, May 22). Vegetarian Dietary Patterns and Cardiovascular Disease. Progress in cardiovascular diseases. https://pubmed.ncbi.nlm.nih.gov/29800598/.

Ke, C., Stukel, T. A., Shah, B. R., Lau, E., Ma, R. C., So, W.-Y., Kong, A. P., Chow, E., Chan, J. C. N., \& Luk, A. (2020, September 18). Age at diagnosis, glycemic trajectories, and responses to oral glucose-lowering drugs in type 2 diabetes in Hong Kong: A population-based observational study. PLOS Medicine. https://journals.plos.org/plosmedicine/article?id=10.1371\%2Fjournal.pmed.1003316.

Kim, H., Caulfield, L. E., Garcia-Larsen, V., Steffen, L. M., Coresh, J., \& Rebholz, C. M. (2019, August 20). PlantBased Diets Are Associated With a Lower Risk of Incident Cardiovascular Disease, Cardiovascular Disease Mortality, and All-Cause Mortality in a General Population of Middle-Aged Adults. Journal of the American Heart Association. https://www.ahajournals.org/doi/epub/10.1161/JAHA.119.012865.

Lee, E. Y., \& Yoon, K.-H. (2018, October 2). Epidemic obesity in children and adolescents: Risk factors and prevention. Frontiers of Medicine. https://link.springer.com/article/10.1007/s11684-018-0640-1.

Li, J., \& Hooker, N. H. (2010, February 8). Childhood obesity and schools: evidence from the national survey of children's health. The Journal of School Health. https://pubmed.ncbi.nlm.nih.gov/20236408/. 
Macknin, M., Kong, T., Weier, A., \& Tang, A. (2015, February 11). Plant-Based, No-Added-Fat or American Heart Association Diets: Impact on Cardiovascular Risk in Obese Children with Hypercholesterolemia and Their Parents. The Journal of Pediatrics. https:/www.jpeds.com/article/S0022-3476(14)01227-X/fulltext.

Patel, K. J., Strait, K. M., Hildebrand, D. A., Amaya, L. L., \& Joyce, J. M. (2020, August 21). Variability in Dietary Quality of Elementary School Lunch Menus with Changes in National School Lunch Program Nutrition Standards. OUP Academic. https://academic.oup.com/cdn/article/4/9/nzaa138/5895317.

Sabate, J., \& Wien, M. (2010, March 17). Vegetarian diets and childhood obesity prevention. The American Journal of Clinical Nutrition. https://pubmed.ncbi.nlm.nih.gov/20237136/.

Satija, A., Bhupathiraju, S. N., Rimm, E. B., Spiegelman, D., Chiuve, S. E., Borgi, L., Willett, W. C., Manson, J. A. E., Sun, Q., \& Hu, F. B. (2016, June 14). Plant-Based Dietary Patterns and Incidence of Type 2 Diabetes in US Men and Women: Results from Three Prospective Cohort Studies. PLOS Medicine. https://journals.plos.org/plosmedicine/article?id=10.1371\%2Fjournal.pmed.1002039.

Satija, A., Bhupathiraju, S. N., Spiegelman, D., Chiuve, S. E., Manson, J. A. E., Willett, W., Rexrode, K. M., Rimm, E. B., \& Hu, F. B. (2017, July 25). Healthful and Unhealthful Plant-Based Diets and the Risk of Coronary Heart Disease in U.S. Adults. Journal of the American College of Cardiology. https://www.ncbi.nlm.nih.gov/pmc/articles/PMC5555375/.

Schanzenbach, D. (2009, April). Do School Lunches Contribute to Childhood Obesity. The Journal of Human Resources. https://www.sesp.northwestern.edu/docs/publications/982412224551ec93458609.pdf.

USDA (2019, July 1). About FNS. USDA. https://www.fns.usda.gov/about-fns\#: :text=Working\%20with\%20our\%20public\%2C\%20private,agriculture\%20and\%20inspires\%20public\%20confidence.

USDA. (2019, April 17). Crediting Tempeh in the Child Nutrition Programs. USDA. https://www.fns.usda.gov/cn/crediting-tempeh-child-nutrition-programs.

USDA. (2016, August 8). Crediting Tofu and Soy Yogurt Products in the School Meal Programs and the CACFP. USDA. https://www.fns.usda.gov/cn/crediting-tofu-and-soy-yogurt-products-school-meal-programs-and-cacfp.

USDA. (2020). Home: Dietary Guidelines for Americans. Home | Dietary Guidelines for Americans. https://dietaryguidelines.gov/.

USDA. (2021, May 18). Lunch Meal Pattern. National School Lunch Program Meal Requirements. https://fnsprod.azureedge.net/sites/default/files/resource-files/NSLP\%20SBP\%20Meal\%20Requirements\%20QA-A2.pdf.

USDA (2020, October 1). National School Lunch Program. USDA ERS - National School Lunch Program. https:/www.ers.usda.gov/topics/food-nutrition-assistance/child-nutrition-programs/national-school-lunch-program.aspx.

Wang, Y., \& Beydoun, M. A. (2009, June). Meat consumption is associated with obesity and central obesity among US adults. International journal of obesity (2005). https://www.ncbi.nlm.nih.gov/pmc/articles/PMC2697260/.

Ward, Z. J., Long, M. W., Resch, S. C., \& Giles, C. M. (2021, May 5). Simulation of Growth Trajectories of Childhood Obesity into Adulthood: NEJM. New England Journal of Medicine. https:/www.nejm.org/doi/full/10.1056/NEJMoa1703860. 
WebMD. (2015, January 16). Food Calculator: Carbs, Calories, Fat, Protein and More. WebMD. https://www.webmd.com/diet/healthtool-food-calorie-counter. 